\title{
Carotid body potentiation during chronic intermittent hypoxia: implication for hypertension
}

\author{
Rodrigo Del Rio ${ }^{1,2}$, Esteban A. Moya ${ }^{1}$ and Rodrigo Iturriaga ${ }^{1 *}$ \\ ${ }^{1}$ Laboratorio de Neurobiología, Departamento Fisiología, Facultad de Ciencias Biológicas, Pontificia Universidad Católica de Chile, Santiago, Chile \\ ${ }^{2}$ Laboratory of Cardiorespiratory Control, Center of Biomedical Research, Universidad Autónoma de Chile, Santiago, Chile
}

\section{Edited by:}

Thiago S. Moreira, University of Sao

Paulo, Brazil

\section{Reviewed by:}

Luciano Drager, University of Sao

Paulo Medical School, Brazil

Thiago S. Moreira, University of Sao

Paulo, Brazil

\section{*Correspondence:}

Rodrigo Iturriaga, Laboratorio de Neurobiología, Departamento

Fisiología, Facultad de Ciencias

Biológicas, Pontificia Universidad

Católica de Chile, Alameda 340,

Santiago 8331150, Chile

e-mail: riturriaga@bio.puc.cl
Autonomic dysfunction is involved in the development of hypertension in humans with obstructive sleep apnea, and animals exposed to chronic intermittent hypoxia $(\mathrm{ClH})$. It has been proposed that a crucial step in the development of the hypertension is the potentiation of the carotid body (CB) chemosensory responses to hypoxia, but the temporal progression of the $\mathrm{CB}$ chemosensory, autonomic and hypertensive changes induced by $\mathrm{ClH}$ are not known. We tested the hypothesis that $\mathrm{CB}$ potentiation precedes the autonomic imbalance and the hypertension in rats exposed to $\mathrm{ClH}$. Thus, we studied the changes in CB chemosensory and ventilatory responsiveness to hypoxia, the spontaneous baroreflex sensitivity (BRS), heart rate variability (HRV) and arterial blood pressure in pentobarbital anesthetized rats exposed to $\mathrm{CIH}$ for 7,14 , and 21 days. After 7 days of $\mathrm{ClH}, \mathrm{CB}$ chemosensory and ventilatory responses to hypoxia were enhanced, while BRS was significantly reduced by 2 -fold in $\mathrm{CIH}$-rats compared to sham-rats. These alterations persisted until 21 days of $\mathrm{ClH}$. After 14 days, $\mathrm{ClH}$ shifted the HRV power spectra suggesting a predominance of sympathetic over parasympathetic tone. In contrast, hypertension was found after 21 days of $\mathrm{ClH}$. Concomitant changes between the gain of spectral HRV, BRS, and ventilatory hypoxic chemoreflex showed that the $\mathrm{CIH}$-induced BRS attenuation preceded the HRV changes. $\mathrm{ClH}$ induced a simultaneous decrease of the BRS gain along with an increase of the hypoxic ventilatory gain. Present results show that $\mathrm{ClH}$-induced persistent hypertension was preceded by early changes in $\mathrm{CB}$ chemosensory control of cardiorespiratory and autonomic function.

\section{INTRODUCTION}

Obstructive sleep apnea (OSA), a highly prevalent sleep-breathing disorder is recognized as an independent risk factor for systemic diurnal hypertension (Gozal and Kheirandish-Gozal, 2008; Somers et al., 2008; Dempsey et al., 2010). Repeated episodes of partial or complete obstruction of the upper airways in OSA leads to intermittent hypoxia and hypercapnia, sleep fragmentation and arousal. The resulting hypoxia and hypercapnia stimulate the carotid body $(\mathrm{CB})$ chemoreceptor's eliciting ventilatory, sympathetic and cardiovascular reflex responses. Remarkably, among all the disturbances produced by OSA, the chronic intermittent hypoxia $(\mathrm{CIH})$ is considered the main factor for the development of hypertension (Gozal and Kheirandish-Gozal, 2008; Somers et al., 2008; Dempsey et al., 2010). Although the link between OSA and diurnal persistent hypertension is well established, the mechanisms responsible for the hypertension are not entirely known. Oxidative stress, inflammation and increased sympathetic outflow have been proposed to mediate the OSA-induced hypertension (Lavie, 2003; Gozal and Kheirandish-Gozal, 2008; Somers et al., 2008). The pathogenic mechanisms involved in the development of hypertension have been extensively studied in animal models of $\mathrm{CIH}$, which simulate the hypoxic-reoxygenation cycles observed in OSA patients and reproduce the main cardiovascular
OSA features (Fletcher et al., 1992; Prabhakar et al., 2005; Iturriaga et al., 2009; Pack and Gislason, 2009).

Studies performed by Fletcher et al. (1992) showed that the denervation of the CBs prevents the development of hypertension in rats exposed to $\mathrm{CIH}$. Despite the findings reported by Fletcher et al. (1992) suggesting the contribution of the CB in the $\mathrm{CIH}$-induced hypertension, the proposal that the peripheral CB chemoreceptor plays a key role in the progression of the hypertension did not receive much attention. However, a growing body of new evidence suggests that an enhanced $\mathrm{CB}$ chemosensory afferent responsiveness to hypoxia contributes to the OSA-induced hypertension (Peng et al., 2003; Rey et al., 2004; Dick et al., 2007; Smith and Pacchia, 2007; Weiss et al., 2007; Iturriaga et al., 2009; Marcus et al., 2010; Prabhakar and Semenza, 2012). Indeed, enhanced sympathetic and cardiorespiratory responses to acute hypoxia in OSA patients have been attributed to a heightened CB chemoreflex drive (Narkiewicz et al., 1998a, 1999; Lusina et al., 2006). In addition, studies performed in animals exposed to $\mathrm{CIH}$ have shown that $\mathrm{CIH}$ selectively enhances $\mathrm{CB}$ chemosensory and ventilatory responses to hypoxia (Peng et al., 2003; Peng and Prabhakar, 2004; Rey et al., 2004; Iturriaga et al., 2009; Del Rio et al., 2010) and produces long-term facilitation of motor ventilatory activity (Mitchell et al., 
2001; McGuire et al., 2003). Recordings from the carotid sinus nerve confirmed that $\mathrm{CIH}$ enhances chemosensory discharge evoked by acute hypoxia (Peng et al., 2003; Peng and Prabhakar, 2004; Rey et al., 2008; Del Rio et al., 2010). Moreover, Peng et al. (2003) found that the CB discharge in normoxia was higher in rats exposed to $\mathrm{CIH}$, applied during $8 \mathrm{~h}$ for 10 days. Thus, $\mathrm{CIH}$ increases $\mathrm{CB}$ chemosensory discharge in normoxia and enhanced the responses to hypoxia. In addition to the potentiation of the $\mathrm{CB}$ chemosensory response, $\mathrm{CIH}$ induces plasticity of the $\mathrm{CB}$ chemosensory activity manifested as long-term facilitation (LTF). Indeed, Peng et al. (2003) reported that chemosensory excitation can be induced by repetitive acute intermittent hypoxia when animals were pre-conditioned using $\mathrm{CIH}$. Indeed, they reported that following 10 episodes of $12 \% \mathrm{O}_{2}$ for $15 \mathrm{~s}$, interspersed with $5 \mathrm{~min}$ of $95 \% \mathrm{O}_{2}$, the chemosensory discharge measured in anesthetized rats increased with each episode of hypoxia and returned to baseline after terminating the 10th hypoxic episode. However, when rats were previously exposed to $\mathrm{CIH}$ ( $15 \mathrm{~s}$ of $5 \% \mathrm{O}_{2} 8 \mathrm{~h}$ /day) for 10 days, and then submitted to the same pattern of 10 intermittent hypoxic episodes, they observed a large increase in the baseline $\mathrm{CB}$ discharge, which persisted for $60 \mathrm{~min}$ after the end of the hypoxic stimulus.

It has been proposed that the attenuation of the cardiac baroreflex and alterations of heart rate variability (HRV) contribute to the CIH-induced hypertension in OSA patients (Shiomi et al., 1996; Narkiewicz et al., 1998a) and adult animals exposed to CIH (Rey et al., 2004, 2008; Lai et al., 2006; Sica and Zhao, 2006). In an elegant study performed by Lai et al. (2006) they showed that autonomic dysfunction in rats exposed to $\mathrm{CIH}$ are related to $\mathrm{BP}$ rises. Moreover, they found that the rats exposed to $\mathrm{CIH}$ display a marked decreased in baroreflex sensitivity (BRS) and HRV along with augmentation of the ventilatory responses to acute hypoxia (Lai et al., 2006). However, whether these changes were related to $\mathrm{CB}$ potentiation was not studied. Furthermore, there are no integrative studies showing that the $\mathrm{CB}$ chemosensory potentiation heads the autonomic imbalance and/or the development of hypertension induced by $\mathrm{CIH}$. Therefore, we seek to unite under one study if the enhanced $\mathrm{CB}$ chemoreflex drive precedes the autonomic imbalance and the hypertension during $\mathrm{CIH}$. Accordingly, we studied i) the progression of changes in $\mathrm{CB}$ afferent chemosensory activity and ventilatory reflex response in response to several inspired $\mathrm{PO}_{2}$ levels, ii) changes in autonomic control using indirect methods (HRV and BRS) and iii) changes in BP (measured 16-20 h after the end of the last intermittent hypoxic cycle) in rats exposed to 7, 14, and 21 of $\mathrm{CIH}$. In addition, we studied the relation between the changes in HRV and BRS with the augmentation of the CB-mediated ventilatory chemoreflex gain.

\section{MATERIALS AND METHODS ANIMALS}

Experiments were performed on male Sprague-Dawley rats weighting initially $\sim 200 \mathrm{~g}$, fed with standard diet ad libitum and kept on a 12-h light/dark schedule (7:30 a.m. to 7:30 p.m.). The protocol was approved by the Bioethical Committee of the Biological Sciences Faculty, Pontificia Universidad Católica de Chile, and was performed according to the Guide for Care and Use of Laboratory Animals, published by the US National Institute of Health (NIH publication no. 85-23, revised 1996).

\section{CHRONIC INTERMITTENT HYPOXIA}

Unrestrained, freely moving rats housed in individual chambers $(12 \times 35 \mathrm{~cm}, 2.21)$ were exposed to a $\mathrm{CIH}$ protocol consisting of hypoxic cycles of $\mathrm{F}_{\mathrm{I}} \mathrm{O}_{2} 5 \%$ for $20 \mathrm{~s}$, followed by room air for $280 \mathrm{~s}$, applied $12 \mathrm{times} / \mathrm{h}$ for $8 \mathrm{~h} /$ day during 7, 14, and 21 days (Iturriaga et al., 2009; Del Rio et al., 2010, 2011, 2012). Chambers were equipped with a rear $\mathrm{N}_{2}$ inlet to produce periodic hypoxic episodes, and a front air extractor, which enables to recover the normoxic level. A computerized system controls the solenoid valve and the alternating cycles of the extractors. During hypoxic exposure, the extractors were stopped, while rear solenoid valves allow $100 \% \mathrm{~N}_{2}$ flows into the chambers. The $\mathrm{O}_{2}$ level in the chambers was monitored with an oxygen analyzer (Ohmeda 5120, USA). The $\mathrm{CO}_{2}$ in the chamber was maintained low by continuous air extraction. A group of age-matched rats was exposed to sham condition, whereas the $\mathrm{N}_{2}$ gas was replaced by means of flushing equal flow of compress air into the chambers. The room temperature was kept at $23-25^{\circ} \mathrm{C}$ and the intermittent hypoxic and sham patterns were applied from 8:30 a.m. to $4: 30$ p.m.

\section{RECORDINGS OF PHYSIOLOGICAL VARIABLES}

Experiments were performed 16 to $20 \mathrm{~h}$ after the end of the last intermittent hypoxic cycle. Rats were anesthetized with sodium pentobarbitone $(40 \mathrm{mg} / \mathrm{kg}$ I.P.), a condition necessary for the study of CB afferent chemosensory discharges, followed by additional doses when necessary to maintain a level of surgical anesthesia. Rats were placed in supine position and the rectal temperature was maintained at $38.0 \pm 0.5^{\circ} \mathrm{C}$ with a regulated heating pad. The trachea was cannulated for airflow recording, and connected to a pneumotachograph to measure tidal volume $\left(V_{T}\right)$, respiratory frequency $\left(f_{R}\right)$, and minute inspiratory volume $\left(\mathrm{V}_{\mathrm{I}}\right)$ as previously described (Del Rio et al., 2010, 2012). One femoral artery was cannulated with a polyethylene tube, filled with $50 \mathrm{IU} / \mathrm{ml}$ of heparin solution for measuring arterial blood pressure (BP) with a transducer (Statham P23, USA). The mean arterial blood pressure (MABP) from the BP signal. Signals were acquired with an analog-digital system PowerLAB 8SP, calibrated, and analyzed with the Chart 7.1-Pro software (ADInstruments, Australia). We measured the ventilatory responses elicited by several levels of $\mathrm{PO}_{2}$ (5 to $670 \mathrm{mmHg}$ ), maintained until the response was in a semi steady state $(\sim 15-30 \mathrm{~s})$. The $\mathrm{PO}_{2}$ level was achieved by mixing different amounts of $100 \% \mathrm{O}_{2}$ and $100 \% \mathrm{~N}_{2}$. The $\mathrm{O}_{2}$ level of each gas mixture was monitored with an oxygen analyzer (Ohmeda 5120, USA).

\section{HEART RATE SIGNAL ACOUISITION AND PROCESSING}

HRV was assessed following the guidelines from the Task Force of the European Society of Cardiology and the North American Society of Pacing and Electrophysiology (Task Force, 1996). The electrocardiogram (ECG) was measured using the II Einthoven lead. Three stainless steel $22 \mathrm{G}$ needle electrodes were localized in the insertion of the right (G1) and left (GND) front legs, and in the left (G2) rear leg. Accordingly, 10 min of ECG recordings 
were digitally acquired at $2 \mathrm{kHz}$ prior to any maneuver. The ECG was examined for detection of ectopic beats that were manually deleted from the recording and interpolated. The ECG signals were analyzed with the HRV module of the Chart 6.1-Pro software. The power spectrum of R-R interval data was obtained using a Fast Fourier Transform algorithm after application of the Hann window. The spectrum of R-R intervals was assessed using the following frequency bands as previously described in rats (Lai et al., 2006; Del Rio et al., 2010): very-low frequency (VLF): DC-0.04 Hz, low frequency (LF): $0.04-0.6 \mathrm{~Hz}$ and high frequency (HF): $0.6-2.4 \mathrm{~Hz}$. Calculations considered the relative power of the LF and HF powers expressed as normalized units and the LF/HF ratio.

\section{SPONTANEOUS BAROREFLEX SENSITIVITY}

Spontaneous BRS over heart rate was calculated using the spectral method (Pagani et al., 1988; Laude et al., 2004). Briefly, the $\alpha$-coefficient index was calculated to estimate the BRS as the spectral transfer function between heart rate and blood pressure variability. BRS was calculated using the low frequency spectral components of the respective signals since it has been shown that the coupling between RR interval and SBP have a non-baroreflex origin in the high frequency range and leads to results that are not all related to BRS (Parati et al., 2000).

For the interaction analysis, BRS was calculated by the sequence method, up-sequences of four or more heart beats, where the R-R interval changed in the same direction and presented a linear correlation coefficient higher than 0.85 were included in the analysis. The average slope of the regression lines was calculated using the HemoLab software (www.haraldstauss. com) to provide the estimation of the BRS in $\mathrm{ms} / \mathrm{mmHg}$ (Straus et al., 2006).

\section{CAROTID BODY CHEMOSENSORY RECORDINGS}

The CB chemosensory discharge was measured as previously described (Iturriaga et al., 2009; Del Rio et al., 2010, 2011, 2012). Briefly, one carotid sinus nerve was dissected and placed on a pair of platinum electrodes and covered with warm mineral oil. The neural signal was pre-amplified (Grass P511, USA), filtered $(30-500 \mathrm{~Hz})$ and fed to an electronic spike-amplitude window discriminator allowing the selection of action potentials of given amplitude above the noise. The lower level of detection was setup when rats breathed $100 \%$ O2 (Dejours Test) and the upper level of the windows was leave open. The selected potentials were counted with a frequency meter to assess the $\mathrm{CB}$ chemosensory frequency of discharge $\left(f_{\mathrm{x}}\right)$, expressed in Hz. Signals were acquired with an analog-digital system PowerLAB 8SP and analyzed with the Chart 7-Pro software (ADInstruments, Australia). The chemosensory discharge was measured at several levels of $\mathrm{PO}_{2}$ (5 to $670 \mathrm{mmHg}$ ), maintained until the response was in semi steady state $(\sim 10$ $20 \mathrm{~s}$ ). Rats breathed spontaneously during the experiments. At the end of the experiments, rats were killed by an overdose of sodium pentobarbitone (100 $\mathrm{mg} / \mathrm{kg}$ I.P.).

The CB chemosensory discharges response curves were fitted to the inspiratory $\mathrm{PO}_{2}$, according to the exponential function (Berkenbosch et al., 1991, 1997).

$$
f_{\mathrm{X}}=\operatorname{bas} f_{\mathrm{X}}+G \exp \left(-K / P \mathrm{O}_{2}\right)
$$

In which, $G$ ( $\max$ - bas) represents the overall gain, $K$ is the shape parameter, and bas is the value of ventilation or CB chemosensory discharge measured during hyperoxic challenge. $\mathrm{CB}$ chemosensory frequency of discharge $\left(f_{\mathrm{x}}\right)$, was expressed in $\mathrm{Hz}$.

\section{CAROTID BODY-MEDIATED CHEMOREFLEX DRIVE GAIN}

To measure the $\mathrm{CB}$ afferent activity gain and the ventilatory reflex gain, the oxygen concentration dependent response curves were fitted to the inspiratory $\mathrm{PO}_{2}$, according to the exponential function (Berkenbosch et al., 1991, 1997).

$$
V_{I}=\operatorname{bas} V+G \exp \left(-K / P O_{2}\right)
$$

In which, $G$ ( $\max$ - bas) represents the overall gain, $K$ is the shape parameter, and bas is the value of ventilation measured during hyperoxic challenge. Inspiratory volume $\left(V_{I}\right)$ expresses as\% of normoxia.

\section{STATISTICAL ANALYSIS}

The data was expressed as mean's \pm SE. Paired comparisons between two groups were performed with the Student $T$-test, and differences between 3 or more groups were assessed with one or Two-Way ANOVA tests, followed by Newman-Keuls posthoc comparisons. All analyses were done with the statistical significance set at $P<0.05$.

\section{RESULTS}

\section{EFFECTS OF CIH ON BASELINE PHYSIOLOGICAL VARIABLES}

CIH produced a significant increase in BP $(P<0.01)$ after 21 days, but not after 7 or 14 days of CIH (Table 1). Indeed, MABP measured 16-20 h after the last hypoxic episode was significantly higher $(P<0.001)$ in rats exposed to $\mathrm{CIH}$ for 21 days $(137.8 \pm 3.0 \mathrm{mmHg}, n=16)$ than in Sham animals $(103.2 \pm$ $3.5 \mathrm{mmHg}, n=11)$. No changes in heart rate $\left(\mathrm{H}_{\mathrm{R}}\right)$ were found among groups. Ventilatory $\left(\mathrm{V}_{\mathrm{T}}, \mathrm{FR}\right.$ and $\left.\mathrm{V}_{\mathrm{I}}\right)$ measurements taken during normoxia $\left(\mathrm{PO}_{2} \sim 150 \pm 10 \mathrm{~mm} \mathrm{Hg}\right)$ from $\mathrm{CIH}$-rats were not significantly different compared to the values obtained from Sham animals (See Table 1). However, CB chemosensory discharges measured in normoxia at the beginning of the recordings,

Table 1 | Changes in physiological variables induced by $\mathrm{ClH}$ in rats.

\begin{tabular}{lcccc}
\hline & Sham & CIH 7 days & CIH 14 days & ClH 21 days \\
\hline MABP $(\mathrm{mmHg})$ & $103.2 \pm 3.5$ & $97.9 \pm 3.5$ & $103.0 \pm 4.2$ & $137.8 \pm 3.0^{* *}$ \\
$\mathrm{H}_{\mathrm{R}}(1 / \mathrm{min})$ & $426.7 \pm 16.9$ & $413.6 \pm 16.2$ & $419.8 \pm 16.8$ & $402.9 \pm 13.9$ \\
$\mathrm{~V}_{\mathrm{T}}(\mathrm{ml} / \mathrm{kg})$ & $4.4 \pm 0.5$ & $3.5 \pm 0.3$ & $3.1 \pm 0.3$ & $3.6 \pm 0.4$ \\
$\mathrm{f}_{\mathrm{R}}(1 / \mathrm{min})$ & $73.5 \pm 5.6$ & $82.4 \pm 7.6$ & $86.9 \pm 10.5$ & $73.5 \pm 3.4$ \\
$\dot{V}(\mathrm{ml} / \mathrm{min} \mathrm{kg})$ & $331.5 \pm 48.0$ & $280.2 \pm 25.6$ & $252.3 \pm 57.9$ & $272.9 \pm 37.4$ \\
$f_{\mathrm{x}}(\mathrm{Hz})$ & $47.8 \pm 5.6$ & $71.9 \pm 3.7^{*}$ & $81.7 \pm 4.4^{*}$ & $80.7 \pm 11.4^{*}$ \\
Weight gain & $3.6 \pm 0.2$ & $2.5 \pm 0.2^{*}$ & $1.8 \pm 0.3^{* *}$ & $2.3 \pm 0.2^{* *}$ \\
(g/day) & & & & \\
\hline
\end{tabular}

MABP, mean arterial blood pressure; $H_{R}$, heart rate; $V_{T}$, tidal volume; $f_{R}$, respiratory rate; $\dot{V}$, minute inspiratory volume; $f_{x}$, frequency of carotid chemosensory discharges measured in normoxia $\left(\mathrm{PO}_{2} \sim 150 \pm 10 \mathrm{~mm} \mathrm{Hg}\right) .{ }^{*} P<0.05 ;{ }^{* *} P<$ $0.01 \mathrm{ClH}$ groups vs. Sham group; Neuman-Keuls post-hoc test after One-Way ANOVA $(n=8-12)$. 
showed a significant 1.5 -fold increase in rats exposed to $\mathrm{CIH}$ for 7,14 , and 21 days compared to the values measured in Sham rats.

\section{CIH-INDUCE EARLY CAROTID BODY CHEMOSENSORY POTENTIATION AND INCREASE THE VENTILATORY CHEMOREFLEX DRIVE}

The CB chemosensory response to acute hypoxia markedly increased after 7 days of $\mathrm{CIH}$, effect that persists following 14 and 21 days of $\mathrm{CIH}$ exposure. The effects of $\mathrm{CIH}$ on $f_{\mathrm{x}}$ at several $\mathrm{PO}_{2}$ levels (from 5 to $670 \mathrm{mmHg}$ ) are summarized in Figure 1. The Two-Way ANOVA analysis showed that the overall chemosensory curves for $\mathrm{PO}_{2}$ were different in $\mathrm{CIH}$-rats exposed to 7, 14, and 21 days of CIH $(P<0.01)$ compared to the curve obtained in Sham rats. The Newman-Keuls test showed that CB chemosensory discharge was higher $(P<0.01)$ in the normoxic and hypoxic range in rats exposed to 7, 14, and 21 days of CIH. Accordingly, rats exposed to $\mathrm{CIH}$ displayed enhanced ventilatory responses $\left(\mathrm{V}_{\mathrm{I}}\right)$ to acute hypoxia. Figure 2 shows the effects of $\mathrm{CIH}$ on $\mathrm{V}_{\mathrm{I}}$ at several inspiratory $\mathrm{PO}_{2}$ levels ( 5 to $670 \mathrm{mmHg}$ ). The overall curves for the relationship between $\mathrm{V}_{\mathrm{I}}$ and $\mathrm{PO}_{2}$ were significantly different at 7, 14, and 21 days of $\mathrm{CIH}$, compared with the curve obtained in Sham rats $(P<0.01$, Two-Way ANOVA). Thus, 7 days of $\mathrm{CIH}$ were enough to potentiate the reflex ventilatory responses to hypoxia, effect that persisted until the day 21 of $\mathrm{CIH}$ exposure. Moreover, the gain of the $\mathrm{CB}$-mediated ventilatory reflex response to acute hypoxia was enhanced by $\mathrm{CIH}$ (Figure 2B).

\section{CIH REDUCE THE BAROREFLEX SENSITIVITY AND SHIFT HEART RATE VARIABILITY}

Baroreflex sensitivity was significantly impaired by CIH. Indeed, $\mathrm{CIH}$-treated rats displayed a reduce BRS compared to Sham animals (Figure 3). We found that $\mathrm{CIH}$-treated rats after 7 days display a marked reduction in the BRS compared to the

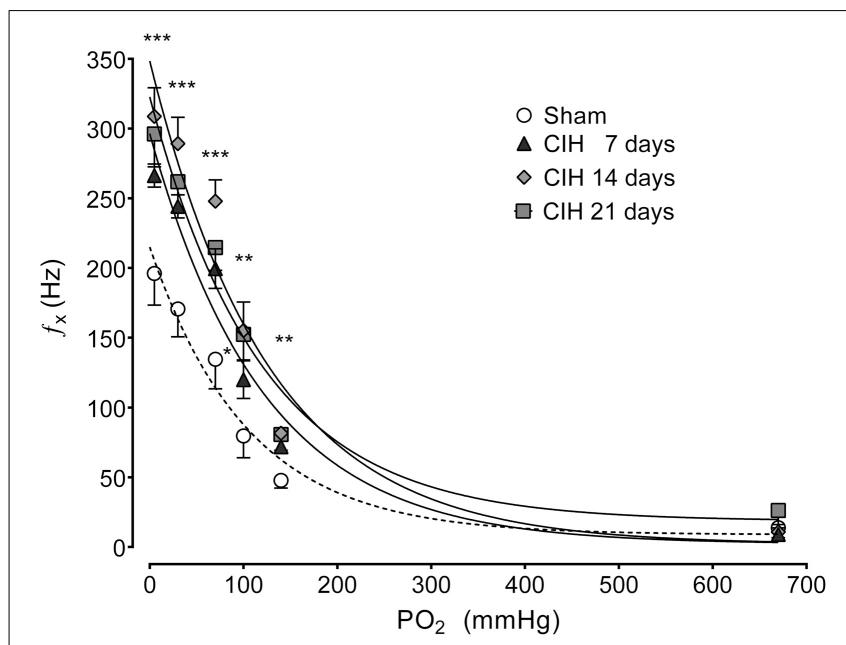

FIGURE 1 | Effects of CIH on CB chemosensory responses to several levels of inspired $\mathbf{P O}_{\mathbf{2}} . f_{X}$, Carotid chemosensory frequency of discharge in $\mathrm{Hz}$. ${ }^{* *} P<0.001,{ }^{* *} P<0.01,{ }^{*} P<0.05$ with sham condition.

Newman-Keuls test after Two-Way ANOVA. $n=6-8$ rats in each group. The chemosensory discharge was fitted to the inspired $\mathrm{PO}_{2}$ using an exponential fit [see Materials and Methods $f_{\mathrm{x}}=$ bas $f_{\mathrm{x}}+G \exp \left(-K / P O_{2}\right)$ ]. The coefficients of correlation for the different curves were: Sham $r=0.82, \mathrm{ClH}$ 7 days $r=0.94, \mathrm{ClH} 14$ days $r=0.92$ and $\mathrm{ClH} 21$ days $r=0.85$. $P<0.01$. values obtained in sham rats and that BRS values remained reduced until the day 21 of exposure to $\mathrm{CIH}(P<0.01$, OneWay ANOVA). Rats exposed to $\mathrm{CIH}$ showed autonomic dysfunction characterized by shifts in the HRV power spectrum. Figure 4 shows representative power spectrum analyses of R$\mathrm{R}$ variability in one Sham rat and rats exposed to $\mathrm{CIH}$ for 7 , 14, and 21 days, respectively. Indeed, rats exposed to 14 and 21 days to $\mathrm{CIH}$ displayed marked changes in the distribution of the relative spectral components of HRV with significantly higher $\mathrm{LF} / \mathrm{HF}$ ratio $(P<0.05$ One-Way ANOVA, $n=8-12)$ as compared to rats exposed to $\mathrm{CIH}$ for 7 days or Sham rats (Figure 5).

\section{INTERACTIONS BETWEEN THE VENTILATORY CHEMOREFLEX GAIN, BAROREFLEX SENTIVITY AND HEART RATE VARIABILITY FOLLOWING CIH}

Figure 6A show the interaction between the spectral LF/HF index and BRS. The CIH-induced attenuation of BRS preceded
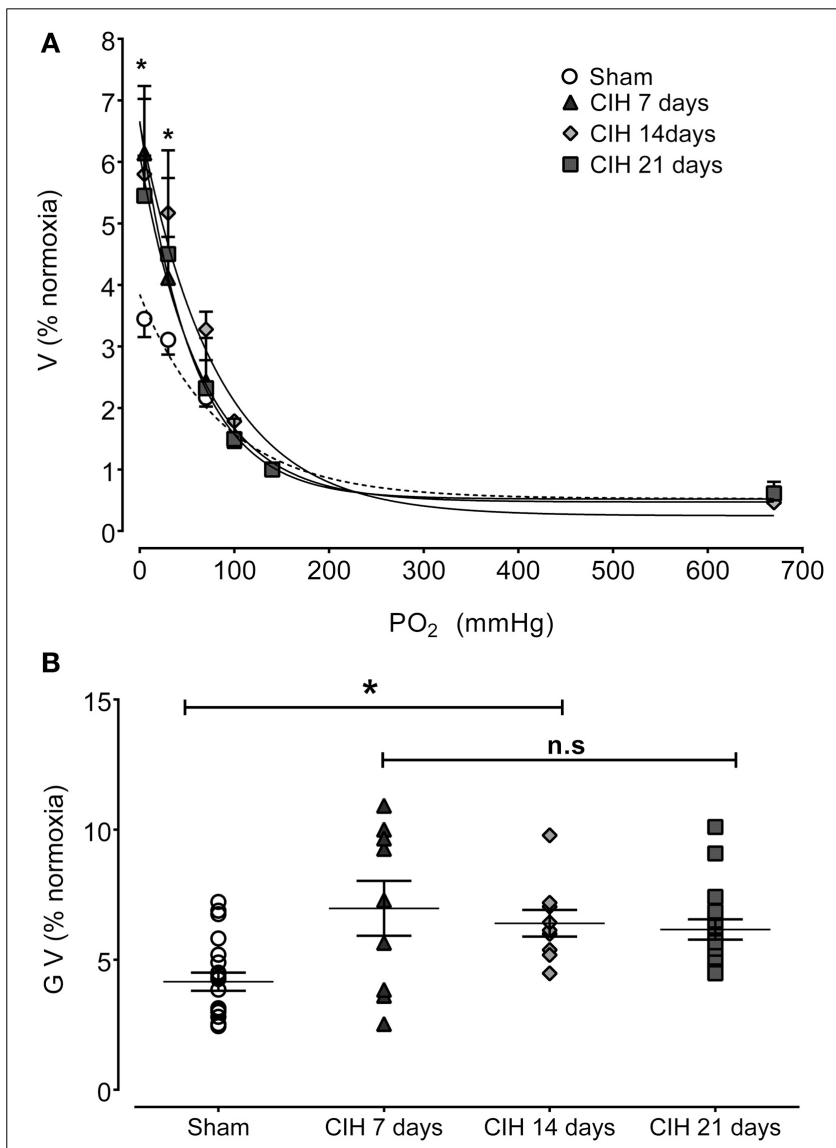

FIGURE 2 | Effects of $\mathrm{CIH}$ on reflex ventilatory minute volume in response to several levels of inspired $\mathbf{P O}_{\mathbf{2}}$. (A) Overall ventilatory curves. (B) Hypoxic ventilatory gain ( $\mathrm{G} \vee$ as \% baseline) corresponding to individual values. Ventilation expressed as percentage of normoxia ventilatory responses. ${ }^{*} P<0.01$ with sham condition. Newman-Keuls test after Two-Way ANOVA in (A) and One-Way ANOVA in (B). $n=8-10$ rats in each group. Ventilation was fitted to the inspired $\mathrm{PO}_{2}$ using an exponential fit [see Materials and Methods $V_{1}=b a s V+G \exp \left(-K / P O_{2}\right)$ ]. The coefficients of correlation for the different curves were: Sham $r=0.82, \mathrm{ClH}$ 7 days $r=0.77, \mathrm{ClH} 14$ days $r=0.91$ and $\mathrm{ClH} 21$ days $r=0.86$. $P<0.01$. 
the changes in the LF/HF spectral index of HRV. Indeed, BRS decreased after 7 days of $\mathrm{CIH}$ without a large change in the LF/HF ratio, but after 14 days the LH/HF index was significant higher. The co-variation of the gain of the CBmediated ventilatory chemoreflex and LF/HF was characterized

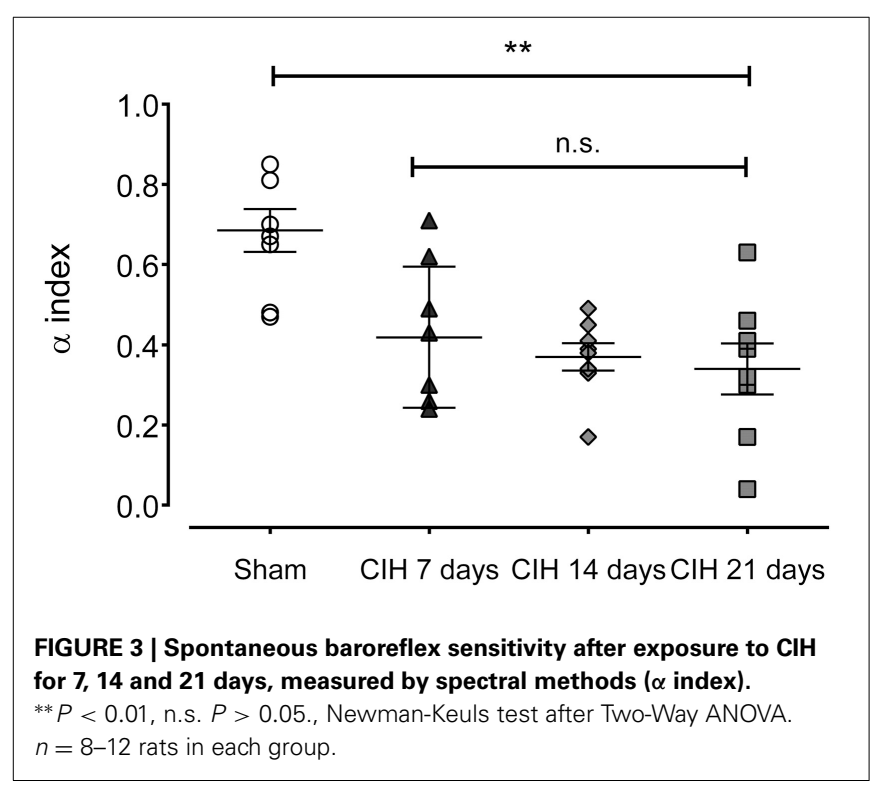

by a small increased in the ventilatory gain at 7 days of $\mathrm{CIH}$ exposure, followed by further increases of the LF/HF at 14 and 21 days of $\mathrm{CIH}$ (Figure 6B). Finally, the analysis of the BRS and the CB-mediated ventilatory chemoreflex gain showed that $\mathrm{CIH}$ induced a concomitant decrease of the BRS along with the increase in the ventilatory gain during the $\mathrm{CIH}$ exposure (Figure 6C).

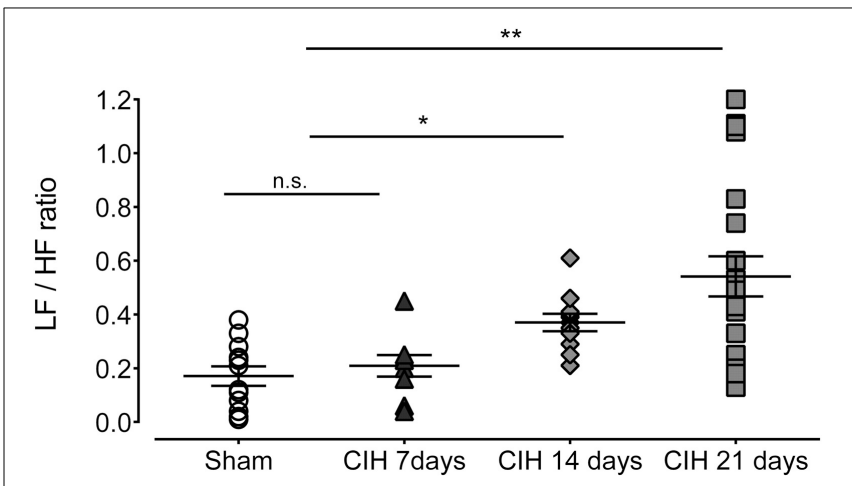

FIGURE 5 | Heart rate variability (HRV) in rats exposed to CIH for 7, 14, and 21 days. LF, low frequency band; HF, high frequency band.

${ }^{* *} P<0.01,{ }^{*} P<0.05$, n.s. $P>0.05$, Newman-Keuls test after Two-Way ANOVA. $n=8-12$ rats in each group.

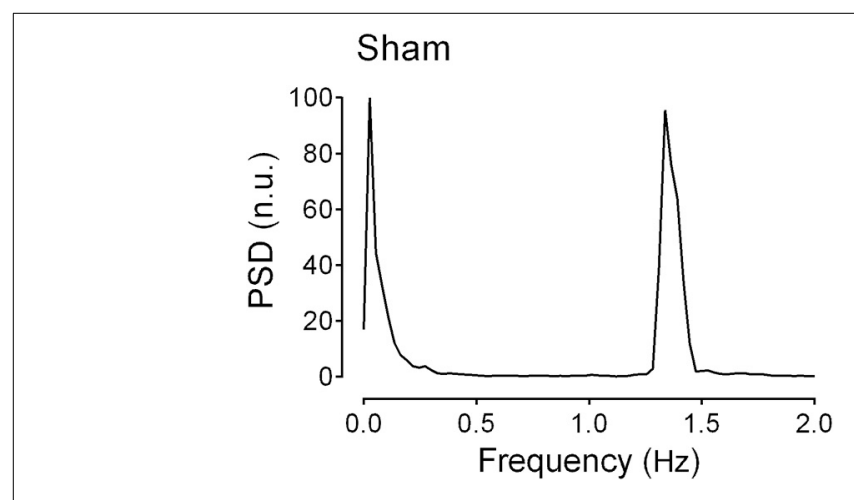

$\mathrm{CIH} 14$ days

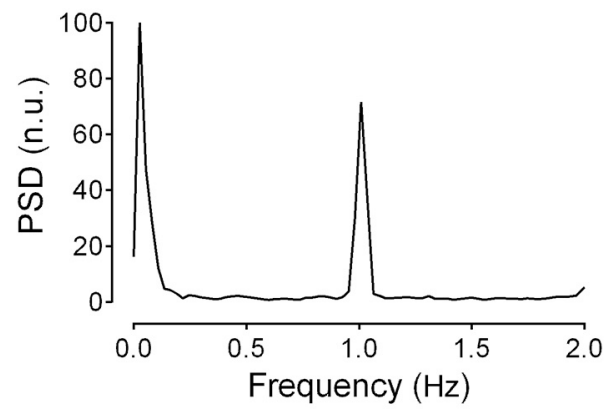

FIGURE 4 | Representative traces of the power spectral density of heart rate variability in one sham rat (Sham), one rat exposed to $\mathrm{ClH}$ for 7 days (CIH 7 ), one rat exposed to $\mathrm{ClH}$

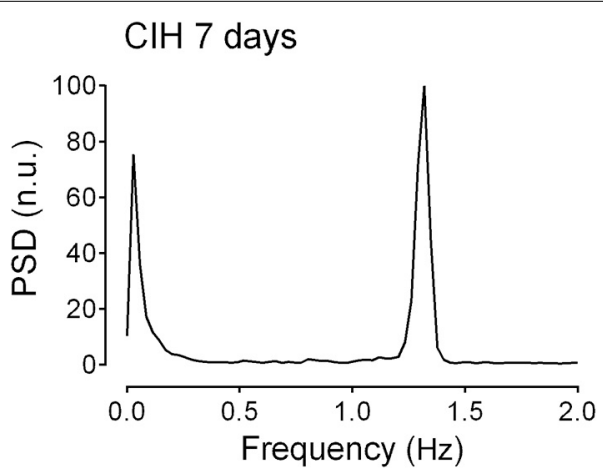

$\mathrm{CIH} 21$ days

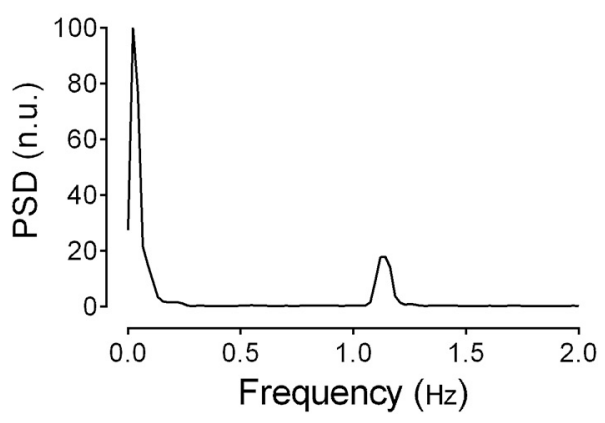

for 14 days (CIH 14) and one rat exposed to $\mathrm{ClH}$ for 21 days (CIH 21). PSD, Power spectral density expressed in normalized units (n.u.). 


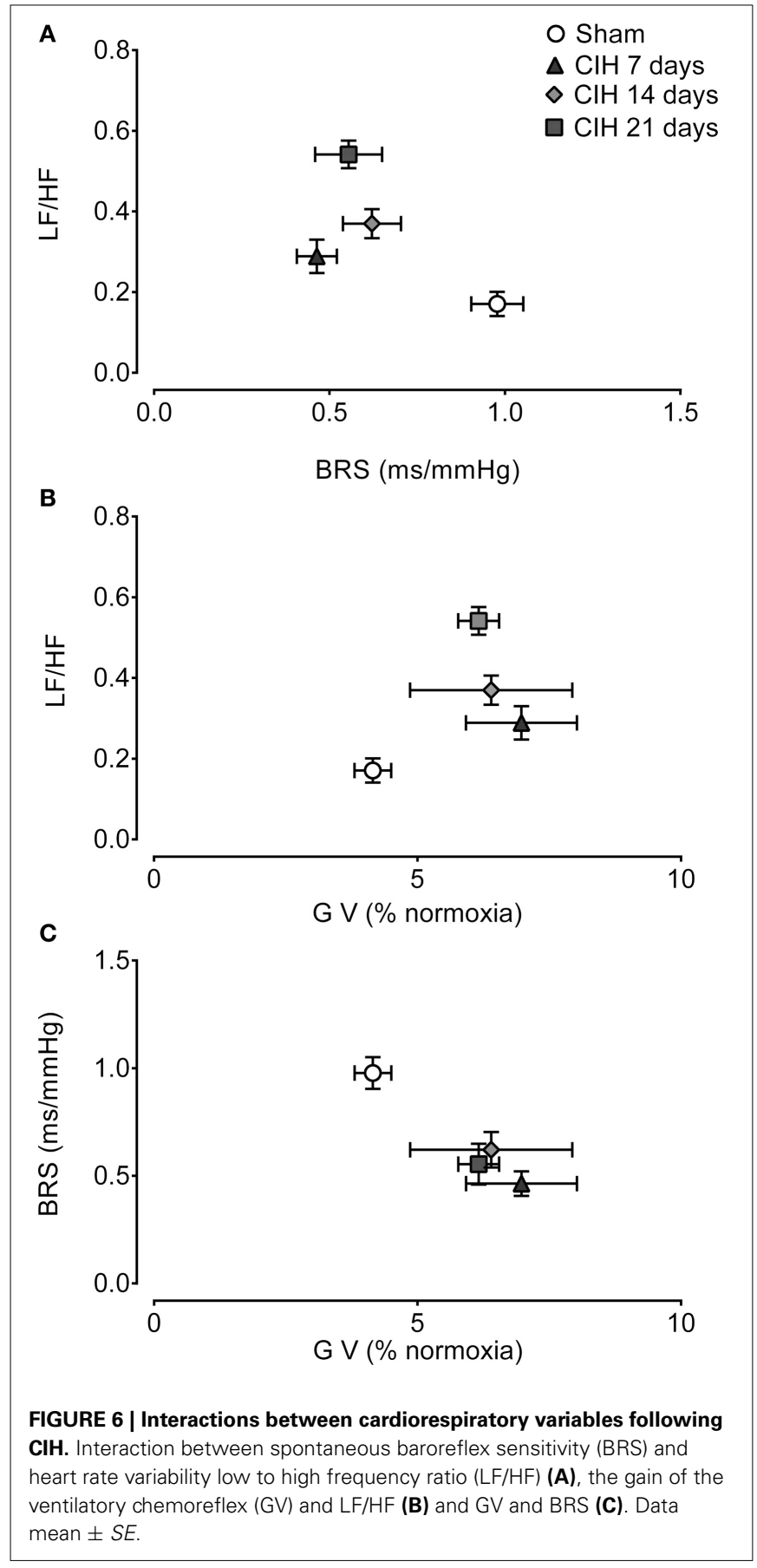

\section{DISCUSSION}

Present results provide a coherent and integrative explanation of the role played by the enhanced $\mathrm{CB}$ chemosensory responses to hypoxia in the progression of the cardiorespiratory alterations and the hypertension induced by $\mathrm{CIH}$, measured 18-24 h after the last intermittent hypoxic stimuli. The main findings of this study show that exposure of rats to cyclic episodes of CIH initially enhance the CB afferent chemosensory drive, decrease BRS and modified the HRV power spectrum toward a predominant sympathetic tone, and finally led to persistent hypertension. Thus, our results support the idea that the persistent hypertension induced by $\mathrm{CIH}$ is preceded by changes in $\mathrm{CB}$ chemosensory. The potentiation of $\mathrm{CB}$ chemosensory and reflex ventilatory responses to hypoxia, as well as the BRS and HRV alterations persisted when rats exhibited a significant elevation of BP in the absence of the $\mathrm{CIH}$ stimulus. The novel finding of the present study is that $\mathrm{CIH}$ induced a simultaneous decrease of BRS gain along with the potentiation of $\mathrm{CB}$-mediated chemoreflex drive suggesting that the increases in the $\mathrm{CB}$ afferent activity may modify the sensitivity of the cardiac baroreflex being the final outcome the development of autonomic imbalance and high blood pressure during exposures to $\mathrm{CIH}$.

\section{ROLE OF THE CAROTID BODY IN THE DEVELOPMENT OF HYPERTENSION FOLLOWING CIH}

A growing body of evidence supports the proposal that an abnormal enhanced CB chemoreceptor responsiveness to hypoxia is involved in the generation of the cardiorespiratory alterations and the hypertension in OSA patients and animals exposed to CIH. Patients with recently diagnosed OSA show enhanced ventilatory, sympathetic, and vasopressor responses to acute hypoxia, attributed to a potentiation of the peripheral chemoreflexes (Cistulli and Sullivan, 1994; Narkiewicz et al., 1998b, 1999). Indeed, Narkiewicz et al. (1999) studied the reflex ventilatory, tachycardic, and pressor responses to acute hypoxia in untreated normotensive patients with OSA, and found that hypoxic stimulation produces larger increases in minute ventilation, heart rate, and arterial blood pressure than control subjects. On the contrary, ventilatory, and pressor responses induced by hypercapnia and by the cold pressor test in OSA patients were not different than those observed in control subjects. In addition, Loredo et al. (2001) reported that OSA hypertensive patients present higher basal tidal volumes, suggesting an enhanced peripheral chemoreflex drive. Similarly, animals exposed to $\mathrm{CIH}$ show enhanced hypoxic ventilatory responses to acute hypoxia (Peng et al., 2003; Peng and Prabhakar, 2004; Rey et al., 2004; Iturriaga et al., 2009; Del Rio et al., 2010) and long-term facilitation of the respiratory motor responses (McGuire et al., 2003; Peng et al., 2003).

\section{AUTONOMIC IMBALANCE DURING CIH}

Autonomic dysregulation has been proposed as a plausible mechanism involved in the progression of the hypertension in OSA patients and animals exposed to $\mathrm{CIH}$. Cyclic hypoxicreoxygenation episodes in OSA patients enhance the ventilatory, cardiovascular, and sympathetic responses induced by acute hypoxia (Carlson et al., 1993; Narkiewicz et al., 1998b). Similarly, animals exposed to $\mathrm{CIH}$ show augmented sympathetic outflow and develop systemic hypertension (Greenberg et al., 1999; Dick et al., 2007; Zoccal et al., 2009). In addition, the autonomic dysregulation has been associated with a reduction of the efficiency of the baroreflex control of heart rate and alterations of HRV in OSA patients (Shiomi et al., 1996; Narkiewicz et al., 1998a) and animals exposed to CIH (Rey et al., 2004, 2008; Lai et al., 2006; Sica and Zhao, 2006; Lin et al., 2007). Moreover, it has been shown that $\mathrm{CIH}$ produces parasympathetic withdrawal, which can further worsen the autonomic imbalance (Lin et al., 2007; Yan et al., 2008). 
The spectral analysis of HRV has two major components defined as the low frequency (LF) band related to sympathetic influences, and the high frequency ( $\mathrm{HF}$ ) band related to the vagal influences and respiratory sinus arrhythmia. The LF/HF ratio is used as an index of the sympathovagal balance on heart rate (Task Force, 1996). Normotensive patients with recently diagnosed OSA showed a shift of the HRV spectral indexes toward the LF band, which is associated with increased sympathetic discharges in the peroneal nerve (Narkiewicz et al., 1998b). Lai et al. (2006) found that 5 days of $\mathrm{CIH}$ exposure increase the LF/HF ratio in awake rats. Our results in anesthetized rats exposed to $\mathrm{CIH}$ showed significant changes in HRV after 14 days of $\mathrm{CIH}$, with a relative predominance of the low frequency component, suggesting a predominance of sympathetic modulation of heart rate. Interestingly, the time required to produce hypertension in rats exposed to $\mathrm{CIH}$ seems to be critically dependent on the hypoxic pattern, the severity, and the length of the exposure. Indeed, Fletcher et al. (1992) found an elevated BP in conscious rats after 7 days of $\mathrm{CIH}$ exposure, which was not different at 35 days of $\mathrm{CIH}$ exposure, indicating that the sustained increase in arterial pressure was maximal after 7 days of CIH. Similarly, Sica et al. (2000) and Peng and Prabhakar (2004) found that systolic arterial pressure increases in rats after 10 days of $\mathrm{CIH}$. On the other hand, Allahdadi et al. (2008) using telemetric BP recordings found that $\mathrm{MABP}$ increased in rats after 14 days of exposure to a $\mathrm{CIH}$ pattern consisting in $90 \mathrm{~s}$ of $\mathrm{N}_{2} 100 \%$ followed by a $90 \mathrm{~s}$ of air, for $7 \mathrm{~h}$ per day. Our results showed that $\mathrm{BP}$ recordings obtained from anesthetized rats exposed to $\mathrm{CIH}$ display increases in systolic and diastolic BP after 21 days of $\mathrm{CIH}$. Therefore, future studies are needed to address the effects of the use of several anesthetic agents on the cardiovascular alterations induced by $\mathrm{CIH}$.

\section{CARDIAC BAROREFLEX ALTERATIONS INDUCED BY CIH}

Present results showed an attenuation of BRS, which preceded the changes in HRV and the hypertension. The changes in BRS found in this study suggest that the $\mathrm{CIH}$-induced hypertension is associated with a reduction of the baroreflex gain. Some studies found that OSA produces a reduction in baroreflex sensitivity, which is associated with hypertension in humans (Carlson et al., 1996; Bonsignore et al., 2002) and animals exposed to OSA (Lai et al., 2006; Gu et al., 2007; Lin et al., 2007; Dematteis et al., 2008; Yan et al., 2008; Chalacheva et al., 2013). However, others studies performed in juvenile rats exposed to $\mathrm{CIH}$ for 10 days suggest that the hypertension is not secondary to the reduction in cardiac baroreflex, but to an enhanced respiratory-sympathetic coupling (Zoccal et al., 2009). Furthermore, Yamamoto et al. (2013) provide evidence that $\mathrm{CIH}$ for 7 days, which increases $\mathrm{BP}$, may reset the renal efferent arm of the baroreflex control in rats without changes in the maximum gain. Moreover, Yamamoto et al. (2013) proposed that acute activation of peripheral chemoreceptor with hypoxic challenges could lead to baroreflex resetting. Here we found that $\mathrm{CB}$ chemosensory discharges are enhanced early during $\mathrm{CIH}$ exposure and that changes take place with a concomitant reduction in the BRS gain. Then, it is plausible that chemoreceptors and baroreceptors interact to establish autonomic dysregulation during $\mathrm{CIH}$.

\section{CHEMOREFLEX AND BAROREFLEX INTERACTION: A LINK TO CIH-INDUCED HYPERTENSION}

Chemoreflexes and baroreflexes play a main role in the control of the cardiorespiratory function in health and disease. Several studies reported interactions between peripheral chemoreceptors and arterial baroreceptors (Heistad et al., 1975; Mancia et al., 1976; Somers et al., 1991). It has been shown that hypoxic chemoreflex activation elicits baroreflex inhibition mainly attributed to central nervous system modulation at the level of the nucleus of the solitary tract (Miura and Reis, 1972). The available evidence suggests that an enhanced CB chemoreflex drive contributes to the cardiorespiratory alteration induced by CIH (Mitchell et al., 2001; McGuire et al., 2003; Peng et al., 2003; Peng and Prabhakar, 2004; Rey et al., 2004; Del Rio et al., 2010). Indeed, CIH enhanced CB chemoreceptor response to hypoxia (Peng and Prabhakar, 2004; Rey et al., 2004; Del Rio et al., 2012), which in turn produces sympathetic activation and hypertension. Accordingly, a blunted baroreflex response to the subsequent hypertension during $\mathrm{CIH}$, may contribute to the further deterioration of the reflex control of BP. Indeed, we found that rats exposed to CIH displayed $\mathrm{CB}$ chemosensory potentiation and BRS reduction with the same time-course. Then, our results suggest that repetitive activation of the peripheral hypoxic chemoreflex during $\mathrm{CIH}$ result in alterations of the baroreflex control. Nevertheless, we cannot rule out that exposure to $\mathrm{CIH}$ per se may reduce BRS, or reset the baroreflex set-point to operate at a higher level of BP. Further studies are needed to prove that $\mathrm{CB}$ potentiation leads to baroreflex impairment following $\mathrm{CIH}$.

\section{STUDY LIMITATIONS}

A major limitation of the present study is that experiments were performed under pentobarbital anesthesia. Unfortunately, this is an obligatory and necessary condition for recording carotid sinus nerve chemosensory discharges in spontaneously breathing animals. The fact that ECG and BP data were collected in anesthetized rats is a methodological limitation because anesthesia may influence HRV and BRS. Indeed, is well known that pentobarbitone anesthesia may induce autonomic depression (Yang et al., 1996) and reductions of the BRS (Shimokawa et al., 1998). We maintained a level of surgical anesthesia (stage III, plane 2) ascertained by the absence of withdrawal reflexes to strong pressure on the paws, with persistence of patellar reflexes. Accordingly, the level of anesthesia was the same to all the experimental groups used in our study. A future comprehensive study is needed to establish a comparison between conscious and anesthetized states.

\section{CONCLUSIONS}

In summary, short-term $\mathrm{CIH}$ increases $\mathrm{CB}$ responsiveness to hypoxia, potentiates the reflex ventilatory response to hypoxia, reduces the baroreflex sensitivity and alters the spectral distribution of the HRV toward the sympathetic modulation, without an increase in BP. In addition, we found that $\mathrm{CIH}$ induced a simultaneous decrease in BRS gain along with an increase of the hypoxic reflex ventilatory gain, suggesting that the potentiation of peripheral chemoreflexes induced by $\mathrm{CIH}$ contributes to the autonomic imbalance. Following long-term $\mathrm{CIH}$, the rats became 
hypertensive and still displayed enhanced CB chemosensory and reflex ventilatory responses to acute hypoxia, BRS impairment, and HRV imbalance. Together, our results show that CB afferent activity is potentiated early during the exposure to $\mathrm{CIH}$ and that precedes the onset of hypertension. Therefore, present results further support the role of peripheral carotid chemoreceptors in the context of $\mathrm{CIH}$-induced autonomic dysregulation and hypertension.

\section{ACKNOWLEDGMENTS}

This work was supported by grant 1100405 from the National Fund for Scientific and Technological Development of Chile (FONDECYT) and Project Puente/28 PUC.

\section{REFERENCES}

Allahdadi, K. J., Cherng, T. W., Pai, H., Silva, A. Q., Walker, B. R., Nelin, L. D., et al. (2008). Endothelin type A receptor antagonist normalizes blood pressure in rats exposed to eucapnic intermittent hypoxia. Am. J. Physiol. Heart. Circ. Physiol. 295, H434-H440. doi: 10.1152/ajpheart.91477.2007

Berkenbosch, A., Olievier, C. N., DeGoede, J., and Kruyt, E. W. (1991). Effect on ventilation of papaverine administered to the brain stem of the anaesthetized cat. J. Physiol. 443, 457-468.

Berkenbosch, A., Teppema, L. J., Olievier, C. N., and Dahan, A. (1997). Influences of morphine on the ventilatory response to isocapnic hypoxia. Anesthesiology 86, 1342-1349. doi: 10.1097/00000542-199706000-00016

Bonsignore, M. R., Parati, G., Insalaco, G., Marrone, O., Castiglioni, P., Romano, S., et al. (2002). Continuous positive airway pressure treatment improves baroreflex control of heart rate during sleep in severe obstructive sleep apnea syndrome. Am. J. Respir. Crit. Care Med. 166, 279-286. doi: 10.1164/rccm.2107117

Carlson, J. T., Hedner, J. A., Sellgren, J., Elam, M., and Wallin, B. G. (1996). Depressed baroreflex sensitivity in patients with obstructive sleep apnea. Am. J. Respir. Crit. Care Med. 154, 1490-1496. doi: 10.1164/ajrccm.154.5.8912770

Carlson, J. T., Hedner, J., Elam, M., Ejnell, H., Sellgren, J., and Wallin, B. G. (1993). Augmented resting sympathetic activity in awake patients with obstructive sleep apnea. Chest 103, 1763-1768. doi: 10.1378/chest.103.6.1763

Chalacheva, P., Thum, J., Yokoe, T., O’Donnell, C. P., and Khoo, M. C. (2013). Development of autonomic dysfunction with intermittent hypoxia in a lean murine model. Respir. Physiol. Neurobiol. 188, 143-151. doi: 10.1016/j.resp. 2013.06.002

Cistulli, P. A., and Sullivan, C. E. (1994). "Pathology of sleep apnea," in Sleep and Breathing, eds N. A. Saunders and C. E. Sullivan (New York, NY: Marcel Decker), 405-448.

Del Rio, R., Moya, E. A., and Iturriaga, R. (2010). Carotid body and cardiorespiratory alterations in intermittent hypoxia: the oxidative link. Eur. Respir. J. 36, 143-150. doi: 10.1183/09031936.00158109

Del Rio, R., Moya, E. A., Muñoz, C., Arias, P., Court, F. A., and Iturriaga, R. (2011). Chronic intermittent hypoxia-induced vascular enlargement and VEGF upregulation in the rat carotid body is not prevented by antioxidant treatment. Am. J. Physiol. Lung Cell. Mol. Physiol. 301, L702-L711. doi: 10.1152/ajplung.00128.2011

Del Rio, R., Moya, E. A., Parga, M. J., Madrid, C., and Iturriaga, R. (2012). Carotid body inflammation and cardiorespiratory alterations in intermittent hypoxia. Eur. Respir. J. 39, 1492-1500. doi: 10.1183/09031936.00141511

Dematteis, M., Julien, C., Guillermet, C., Sturm, N., Lantuejoul, S., Mallaret, M., et al. (2008). Intermittent hypoxia induces early functional cardiovascular remodeling in mice. Am. J. Respir. Crit. Care Med. 177, 227-235. doi: 10.1164/rccm.200702-238OC

Dempsey, J. A., Veasey, S. C., Morgan, B. J., and O'Donnell, C. P. (2010). Pathophysiology of sleep apnea. Physiol. Rev. 90, 47-112. doi: 10.1152/physrev.00043.2008

Dick, T. E., Hsieh, Y. H., Wang, N., and Prabhakar, N. R. (2007). Acute intermittent hypoxia increases both phrenic and sympathetic nerve activities in the rat. Exp. Physiol. 92, 87-97. doi: 10.1113/expphysiol.2006.035758

Fletcher, E. C., Lesske, J., Behm, R., Miller, C. C., Stauss, H., and Unger, T. (1992). Carotid chemoreceptors, systemic blood pressure, and chronic episodic hypoxia mimicking sleep apnoea. J. Appl. Physiol. 72, 1978-1984.
Gozal, D., and Kheirandish-Gozal, L. (2008). Cardiovascular morbidity in obstructive sleep apnea, oxidative stress, inflammation, and much more. Am. J. Respir. Crit. Care Med. 177, 369-375. doi: 10.1164/rccm.2006081190PP

Greenberg, H. E., Sica, A., Batson, D., and Scharf, S. M. (1999). Chronic intermittent hypoxia increases sympathetic responsiveness to hypoxia and hypercapnia. J. Appl. Physiol. 86, 298-305.

Gu, H., Lin, M., Liu, J., Gozal, D., Scrogin, K. E., Wurster, R., et al. (2007). Selective impairment of central mediation of baroreflex in anesthetized young adult Fischer 344 rats after chronic intermittent hypoxia. Am. J. Physiol. Heart Circ. Physiol. 293, H2809-H2818. doi: 10.1152/ajpheart.00358.2007

Heistad, D. D., Abboud, F. D., Mark, A. L., and Schmid, P. G. (1975). Effect ofbaroreceptor activity on ventilatory response to chemoreceptor stimulation. J. Appl. Physiol. 39, 411-416.

Iturriaga, R., Moya, E. A., and Del Rio, R. (2009). Carotid body potentiation induced by intermittent hypoxia: implications for cardiorespiratory changes induced by sleep apnoea. Clin. Exp. Pharmacol. Physiol. 36, 1197-1204. doi: 10.1111/j.1440-1681.2009.05213.x

Lai, C. J., Yang, C. C. H., Hsu, Y. Y., Lin, Y. N., and Kuo, T. B. J. (2006). Enhanced sympathetic outflow and decreased baroreflex sensitivity are associated with intermittent hypoxia-induced systemic hypertension in conscious rats. J. Appl. Physiol. 100, 1974-1982. doi: 10.1152/japplphysiol.010 51.2005

Laude, D., Elghozi, J. L., Girard, A., Bellard, E., Bouhaddi, M., Castiglioni, P., et al. (2004). Comparison of various techniques used to estimate spontaneous baroreflex sensitivity. The EuroBaVar study. Am. J. Physiol. Regul. Integr. Comp. Physiol. 286, R226-R231. doi: 10.1152/ajpregu.00709.2002

Lavie, L. (2003). Obstructive sleep apnoea syndrome - an oxidative stress disorder. Sleep Med. Rev. 7, 35-51. doi: 10.1053/smrv.2002.0261

Lin, M., Liu, R., Gozal, D., Wead, W. B., Chapleau, M. W., Wurster, R., et al. (2007). Chronic intermittent hypoxia impairs baroreflex control of heart rate but enhances heart rate responses to vagal efferent stimulation in anesthetized mice. Am. J. Physiol. Heart Circ. Physiol. 293, H997-H1006. doi: 10.1152/ajpheart.01124.2006

Loredo, J. S., Clausen, J. L., Nelesen, R. A., Ancoli-Israel, S., Ziegler, M. G., and Dimsdale, J. E. (2001). Obstructive sleep apnea and hypertension: are peripheral chemoreceptors involved? Med. Hypotheses 56, 17-19. doi: 10.1054/mehy.2000.1086

Lusina, S. J., Kennedy, P. M., Inglis, J. T., McKenzie, D. C., Ayas, N. T., and Sheel, A. W. (2006). Long-term intermittent hypoxia increases sympathetic activity and chemosensitivity during acute hypoxia in humans. J. Physiol. 575, 961-970. doi: 10.1113/jphysiol.2006.114660

Mancia, G., Shepherd, J. T., and Donald, D. E. (1976). Interplay among carotid sinus, cardiopulmonary, and carotid body reflexes in dogs. Am. J. Physiol. 230, 19-24.

Marcus, N. J., Li, Y. L., Bird, C. E., Schultz, H. D., and Morgan, B. J. (2010). Chronic intermittent hypoxia augments chemoreflex control of sympathetic activity: role of the angiotensin II type 1 receptor. Respir. Physiol. Neurobiol. 171, 36-45. doi: 10.1016/j.resp.2010.02.003

McGuire, M., Zhang, Y., White, D. P., and Ling, L. (2003). Chronic intermittent hypoxia enhances ventilatory long-term facilitation in awake rats. J. Appl. Physiol. 95, 1499-1508. doi: 10.1152/japplphysiol.00044.2003

Mitchell, G. S., Baker, T. L., Nanda, S. A., Fuller, D. D., Zabka, A. G., Hodgeman, B. A., et al. (2001). Intermittent hypoxia and respiratory plasticity. J. Appl. Physiol. 90, 2466-2475.

Miura, M., and Reis, D. J. (1972). The role of the solitary and paramedian reticular nuclei in mediating cardiovascular reflex responses from carotid baro- and chemoreceptors. J. Physiol. 223, 525-548.

Narkiewicz, K., Montano, N., Cogliati, C., van de Borne, P. J., Dyken, M. E., and Somers, V. K. (1998a). Altered cardiovascular variability in obstructive sleep apnea. Circulation 98, 1071-1077. doi: 10.1161/01.CIR.98.11.1071

Narkiewicz, K., van de Borne, P. J., Montano, N., Dyken, M. E., Phillips, B. G., and Somers, V. K. (1998b). Contribution of tonic chemoreflex activation to sympathetic activity and blood pressure in patients with obstructive sleep apnea. Circulation 97, 943-945. doi: 10.1161/01.CIR.97.10.943

Narkiewicz, K., van de Borne, P. J., Pesek, C. A., Dyken, M. E., Montano, N., and Somers, V. K. (1999). Selective potentiation of peripheral chemoreflex sensitivity in obstructive sleep apnea. Circulation 99, 1183-1189. doi: 10.1161/01.CIR.99.9.1183 
Pack, A. I., and Gislason, T. (2009). Obstructive sleep apnea and cardiovascular disease: a perspective and future directions. Prog. Cardiovasc. Dis. 51, 434-451. doi: 10.1016/j.pcad.2009.01.002

Pagani, M., Somers, V., Furlan, R., Dell'Orto, S., Conway, J., Baselli, G., et al. (1988). Changes in autonomic regulation induced by physical training in mild hypertension. Hypertension 12, 600-610. doi: 10.1161/01.HYP.12.6.600

Parati, G., Di Rienzo, M., and Mancia, G. (2000). How to measure baroreflex sensitivity: from the cardiovascular laboratory to daily life. J. Hypertens. 18, 7-19. doi: 10.1097/00004872-200018010-00003

Peng, Y. J., Overholt, J. L., Kline, D., Kumar, G. K., and Prabhakar, N. R. (2003). Induction of sensory long-term facilitation in the carotid body by intermittent hypoxia: implications for recurrent apneas. Proc. Natl. Acad. Sci. U.S.A. 100, 10073-10078. doi: 10.1073/pnas.1734109100

Peng, Y. J., and Prabhakar, N. R. (2004). Effect of two paradigms of chronic intermittent hypoxia on carotid body sensory activity. J. Appl. Physiol. 96, 1236-1242. doi: 10.1152/japplphysiol.00820.2003

Prabhakar, N. R., Peng, Y. J., Jacono, F. J., Kumar, G. K., and Dick, T. E. (2005). Cardiovascular alterations by chronic intermittent hypoxia: Importance of carotid body chemoreflexes. Clin. Exp. Pharmacol. Physiol. 32, 447-449. doi: 10.1111/j.1440-1681.2005.04209.x

Prabhakar, N. R., and Semenza, G. L. (2012). Adaptive and maladaptive cardiorespiratory responses to continuous and intermittent hypoxia mediated by hypoxia-inducible factors 1 and 2. Physiol. Rev. 92, 967-1003. doi: 10.1152/physrev.00030.2011

Rey, S., Del Rio, R., Alcayaga, J., and Iturriaga, R. (2004). Chronic intermittent hypoxia enhances cat chemosensory and ventilatory responses to hypoxia. J. Physiol. 560, 577-586. doi: 10.1113/jphysiol.2004.072033

Rey, S., Tarvainen, M. P., Karjalainen, P. A., and Iturriaga, R. (2008). Dynamic timevarying analysis of heart rate and blood pressure variability in cats exposed to short-term chronic intermittent hypoxia. Am. J. Physiol. Reg. Integr. Comp. Physiol. 295, R28-R37. doi: 10.1152/ajpregu.00070.2008

Shimokawa, A., Kunitake, T., Takasaki, M., and Kannan, H. (1998). Differential effects of anesthetics on sympathetic nerve activity and arterial baroreceptor reflex in chronically instrumented rats. J. Auton. Nerv. Syst. 72, 46-54. doi: 10.1016/S0165-1838(98)00084-8

Shiomi, T., Guilleminault, C., Sasanabe, R., Hirota, I., Maekawa, M., and Kobayashi, T. (1996). Augmented very low frequency component of heart rate variability during obstructive sleep apnea. Sleep 19, 370-377.

Sica, A. L., Greenberg, H. E., Ruggiero, D. A., and Scharf, S. M. (2000). Chronicintermittent hypoxia: A model of sympathetic activation in the rat. Respir. Physiol. 121, 173-184. doi: 10.1016/S0034-5687(00)00126-2

Sica, A. L., and Zhao, N. (2006). Heart rate variability in conscious neonatal swine: spectral features and responses to short-term intermittent hypoxia. BMC Physiol. 6: 5. doi: 10.1186/1472-6793-6-5

Smith, M. L., and Pacchia, C. F. (2007). Sleep apnoea and hypertension: role of chemoreflexes in humans. Exp. Physiol. 92, 45-50. doi: 10.1113/expphysiol.2006.033753

Somers, V. K., Mark, A. L., and Abboud, F. M. (1991). Interaction of baroreceptor and chemoreceptor reflex control of sympathetic nerve activity in normal humans. J. Clin. Invest. 87, 1953-1957. doi: 10.1172/JCI115221
Somers, V. K., White, D. P., Amin, R., Abraham, W. T., Costa, F., Culebras, A., et al. (2008). Sleep apnea and cardiovascular disease: an american heart association. In collaboration with the national heart, lung, and blood Institute National Center on Sleep Disorders Research (National Institutes of Health). J. Am. Coll. Cardiol. 52, 686-717. doi: 10.1016/j.jacc.2008.05.002

Straus, S. M., Kors, J. A., De Bruin, M. L., van der Hooft, C. S., Hofman, A., Heeringa, J., et al. (2006). Prolonged QTc interval and risk of sudden cardiac death in a population of older adults. J. Am. Coll. Cardiol. 47, 362-367. doi: 10.1016/j.jacc.2005.08.067

Task Force of the European Society of Cardiology and the North American Society of Pacing and Electrophysiology. (1996). Heart rate variability. Standards of measurement, physiological interpretation, and clinical use. Eur. Heart. J. 17, 354-381. doi: 10.1093/oxfordjournals.eurheartj.a014868

Weiss, J. W., Liu, M. D., and Huang, J. (2007). Physiological basis for a causal relationship of obstructive sleep apnoea to hypertension. Exp. Physiol. 92, 21-26. doi: 10.1113/expphysiol.2006.035733

Yamamoto, K., Eubank, W., Franzke, M., and Mifflin, S. (2013). Resetting of the sympathetic baroreflex is associated with the onset of hypertension during chronic intermittent hypoxia. Auton. Neurosci. 173, 22-27. doi: 10.1016/j.autneu.2012.10.015

Yan, B., Soukhova-O'Hare, G. K., Li, L., Lin, Y., Gozal, D., Wead, W. B., et al. (2008). Attenuation of heart rate control and neural degeneration in nucleus ambiguus following chronic intermittent hypoxia in young adult Fischer 344 rats. Neuroscience 153, 709-720. doi: 10.1016/j.neuroscience.2008. 01.066

Yang, C. C. H., Kuo, T. B. J., and Chan, S. H. H. (1996). Auto- and cross-spectral analysis of cardiovascular fluctuations during pentobarbital anesthesia in the rat. Am. J. Physiol. Heart Circ. Physiol. 270, H575-H582.

Zoccal, D. B., Bonagamba, L. G., Paton, J. F., and Machado, B. H. (2009). Sympathetic-mediated hypertension of awake juvenile rats submitted to chronic intermittent hypoxia is not linked to baroreflex dysfunction. Exp. Physiol. 94, 972-983. doi: 10.1113/expphysiol.2009.048306

Conflict of Interest Statement: The authors declare that the research was conducted in the absence of any commercial or financial relationships that could be construed as a potential conflict of interest.

Received: 01 July 2014; accepted: 24 October 2014; published online: 12 November 2014.

Citation: Del Rio R, Moya EA and Iturriaga R (2014) Carotid body potentiation during chronic intermittent hypoxia: implication for hypertension. Front. Physiol. 5:434. doi: 10.3389/fphys.2014.00434

This article was submitted to Integrative Physiology, a section of the journal Frontiers in Physiology.

Copyright (c) 2014 Del Rio, Moya and Iturriaga. This is an open-access article distributed under the terms of the Creative Commons Attribution License (CC BY). The use, distribution or reproduction in other forums is permitted, provided the original author(s) or licensor are credited and that the original publication in this journal is cited, in accordance with accepted academic practice. No use, distribution or reproduction is permitted which does not comply with these terms. 